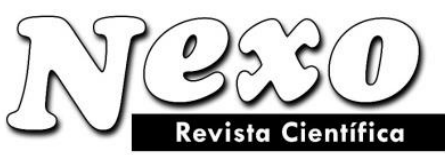

Vol. 32, No. 01, pp. 61-74/Junio 2019

\title{
DISEÑO DE UN INTERCAMBIADOR DE CALOR DE SERPENTÍN PARA EL ENFRIAMIENTO DE ACETONA
}

\section{DESIGN OF HELICAL-COIL HEAT EXCHANGER FOR ACETONE COOLING}

\author{
A. Pérez Sánchez ${ }^{1, *}$, E.J. Pérez Sánchez ${ }^{2}$, A. Heredia Sánchez ${ }^{3}$, L. Pazos Amayuela ${ }^{4}$ \\ ${ }^{1}$ Universidad de Camagüey "Ignacio Agramonte Loynaz”, Facultad de Ciencias Aplicadas, Departamento \\ de Ingeniería Química, Camagüey, Cuba, CP 74650. \\ ${ }^{2}$ Empresa Servicios Automotores S.A., Dirección Comercial, Ciego de Ávila, Cuba. \\ ${ }^{3}$ Universidad de Camagüey "Ignacio Agramonte Loynaz", Departamento de Inversiones, Camagüey, \\ Cuba. \\ ${ }^{4}$ Universidad de Camagüey “Ignacio Agramonte Loynaz”, Facultad de Ciencias Aplicadas, Departamento \\ de Ingeniería Industrial, Camagüey, Cuba. \\ *amaury.psanchez@ reduc.edu.cu
}

(recibido/received: 10-Diciembre-2018; aceptado/accepted: 13-Abril-2019)

\section{RESUMEN}

Los intercambiadores de calor de serpentín son empleados en varias aplicaciones industriales debido a su estructura compacta y los elevados coeficientes de transferencia de calor que usualmente se obtienen. En el presente trabajo se realizó el diseño de un intercambiador de calor de serpentín para llevar a cabo el enfriamiento de una corriente de acetona líquida. El equipo tendrá un coeficiente global de transferencia de calor de $23,88 \mathrm{kcal} / \mathrm{h} . \mathrm{m} 2{ }^{\circ} \mathrm{C}$, un área de transferencia de calor de $6,60 \mathrm{~m} 2$, un número real de vueltas de serpentín igual a 53 y una altura de $2,58 \mathrm{~m}$. Tanto la corriente de acetona como la de agua no sobrepasan la caída de presión máxima establecida por el proceso. También se determinó la influencia que presenta el incremento de tanto el caudal de alimentación como la temperatura de entrada de la acetona sobre cuatro parámetros de diseño del intercambiador de calor de serpentín: coeficiente global de transferencia de calor, área de transferencia de calor, número real de vueltas del serpentín, y altura del equipo, obteniéndose como resultado que el incremento de las dos variables iniciales aumenta los valores a obtener de estos cuatro parámetros. Se utilizaron plantillas de cálculo Excel tanto para la confección de los gráficos como la solución de la metodología de cálculo utilizada en este estudio.

Palabras claves: Acetona, Diseño, Intercambiador de calor, Serpentín.

\section{ABSTRACT}

The helical coil heat exchangers are employed in several applications due to their compact structure and the high heat transfer coefficients usually obtained. In the present work, the design of a helical coil heat exchanger is accomplished in order to carry out the cooling of a liquid acetone stream. The equipment will has an overall heat transfer coefficient of $23.88 \mathrm{kcal} / \mathrm{h} . \mathrm{m} 2{ }^{\circ} \mathrm{C}$; a heat transfer area of $6.60 \mathrm{~m} 2$; an actual 
number of turns of coil equal to 53; and a height of $2.58 \mathrm{~m}$. Both the acetone and the water stream do not exceed the maximum pressure drop established by the process. The influence of increasing both the acetone feed flowrate and inlet temperature on four design parameters of the helical coil heat exchanger: global heat transfer coefficient, heat transfer area, actual number of turns of the coil, and height of the equipment, was also determined, obtaining as a result that the increase of the two initial variables increases the values to be obtained of these four parameters. Excel spreadsheets were used to obtain the graphics and to solve the design methodology used in this study.

Keywords: Acetone, Design, Heat exchanger, Helical coil.

\section{NOMENCLATURA}

\begin{tabular}{|c|c|}
\hline A & Área de transferencia de calor \\
\hline$A_{\text {serp }}$ & Área de sección transversal del serpentín \\
\hline $\mathrm{B}$ & Diámetro exterior del cilindro interior \\
\hline $\mathrm{C}_{\mathrm{A}}$ & Coeficiente de arrastre \\
\hline $\mathrm{Cp}$ & Calor específico \\
\hline $\mathrm{d}_{\mathrm{i}}$ & Diámetro interior del serpentín \\
\hline $\mathrm{d}_{\mathrm{e}}$ & Diámetro exterior del serpentín \\
\hline $\mathrm{D}_{\mathrm{h}}$ & Diámetro promedio de la hélice \\
\hline $\mathrm{D}_{\mathrm{i}}$ & Diámetro interior del cilindro exterior \\
\hline $\mathrm{Di}_{\mathrm{h}}$ & Diámetro interior de la hélice \\
\hline $\mathrm{De}_{\mathrm{h}}$ & Diámetro exterior de la hélice \\
\hline $\mathrm{D}_{\mathrm{eq}}$ & Diámetro equivalente de la coraza \\
\hline $\mathrm{e}$ & Espesor del tubo serpentín \\
\hline $\mathrm{E}$ & Factor \\
\hline$f$ & Facitor de fricción \\
\hline $\mathrm{G}$ & Velocidad másica \\
\hline $\mathrm{h}_{\mathrm{i}}$ & $\begin{array}{l}\text { Coeficiente pelicular de transferencia de calor del agua basado en el diámetro } \\
\text { interior del serpentín para el tubo recto }\end{array}$ \\
\hline$h_{\mathrm{i} 0}$ & $\begin{array}{l}\text { Coeficiente pelicular de transferencia de calor basado en el diámetro exterior } \\
\text { del serpentín }\left(\mathrm{h}_{\mathrm{i} 0}\right)\end{array}$ \\
\hline$h_{\text {ic }}$ & $\begin{array}{l}\text { Coeficiente pelicular de transferencia de calor del agua corregido para el tubo } \\
\text { enroscado }\end{array}$ \\
\hline $\mathrm{h}_{0}$ & $\begin{array}{l}\text { Coeficiente pelicular de transferencia de calor del fluido que circula por la } \\
\text { coraza }\end{array}$ \\
\hline $\mathrm{H}$ & Altura \\
\hline $\mathrm{k}$ & Conductividad térmica \\
\hline $\mathrm{L}_{\text {serp }}$ & Longitud del serpentín \\
\hline $\mathrm{m}$ & Caudal másico \\
\hline MLDT & Media Logarítmica de la Diferencia de Temperatura \\
\hline $\mathrm{n}$ & Número real de vueltas del serpentín \\
\hline $\mathrm{N}$ & Número teórico de vueltas del serpentín \\
\hline $\mathrm{p}$ & Paso \\
\hline $\operatorname{Pr}$ & Número de Prandtl \\
\hline$\Delta \mathrm{P}$ & Caída de presión \\
\hline $\mathrm{q}$ & Caudal volumétrico \\
\hline Q & Calor intercambiado \\
\hline $\mathrm{Re}$ & Número de Reynolds \\
\hline $\mathrm{t}$ & Temperatura del fluido frío \\
\hline$\Delta \mathrm{t}$ & Diferencia de temperatura corregida \\
\hline
\end{tabular}

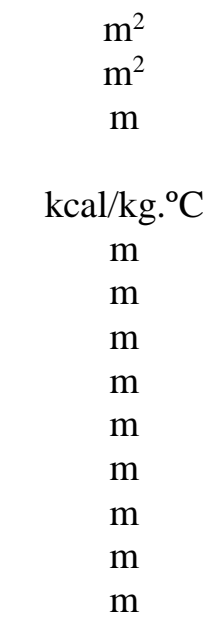

$\mathrm{kg} / \mathrm{m}^{2} \cdot \mathrm{h}$

$\mathrm{kcal} / \mathrm{h} \cdot \mathrm{m} 2 .{ }^{\circ} \mathrm{C}$

$\mathrm{kcal} / \mathrm{h} \cdot \mathrm{m}^{2} .{ }^{\circ} \mathrm{C}$

$\mathrm{kcal} / \mathrm{h} . \mathrm{m}^{2} .{ }^{\circ} \mathrm{C}$

$\mathrm{m}$

$\mathrm{kcal} / \mathrm{h} . \mathrm{m} .{ }^{\circ} \mathrm{C}$

$\mathrm{m}$

$\mathrm{kg} / \mathrm{h}$

${ }^{\circ} \mathrm{C}$

Adimensional

Adimensional

$\mathrm{m}$

Adimensional

$\mathrm{Pa}$

$\mathrm{m}^{3} / \mathrm{h}$

$\mathrm{kcal} / \mathrm{h}$

Adimensional

${ }^{\circ} \mathrm{C}$

${ }^{\circ} \mathrm{C}$ 


\begin{tabular}{|c|c|c|}
\hline $\mathrm{T}$ & Temperatura del fluido caliente & ${ }^{\circ} \mathrm{C}$ \\
\hline $\mathrm{U}$ & Coeficiente global de transferencia de calor & $\mathrm{kcal} / \mathrm{h} \cdot \mathrm{m}^{2}{ }^{\circ} \mathrm{C}$ \\
\hline $\mathrm{v}$ & Velocidad & $\mathrm{m} / \mathrm{s}$ \\
\hline $\mathrm{V}_{\mathrm{a}}$ & Volumen del ánulo & $\mathrm{m}^{3}$ \\
\hline$V_{\text {flujo }}$ & Volumen disponible para el flujo en el ánulo & $\mathrm{m}^{3}$ \\
\hline $\mathrm{V}_{\text {serp }}$ & Volumen ocupado por el serpentín & $\mathrm{m}^{3}$ \\
\hline \multicolumn{3}{|c|}{ Símbolos griegos } \\
\hline$\rho$ & Densidad & $\mathrm{kg} / \mathrm{m}^{3}$ \\
\hline$\mu$ & Viscosidad & $\mathrm{kg} / \mathrm{m} \cdot \mathrm{h}$ \\
\hline \multicolumn{3}{|c|}{ Subíndices } \\
\hline $\mathrm{e}$ & Entrada & \\
\hline $\mathrm{s}$ & Salida & \\
\hline acet & Acetona & \\
\hline agua & Agua & \\
\hline
\end{tabular}

\section{INTRODUCCIÓN}

El intercambio de calor entre fluidos constituye uno de los procesos físicos más importantes utilizados hoy en día en la industria de procesos químicos. Un intercambiador de calor es un dispositivo en donde ocurre la transferencia de energía térmica entre dos fluidos que se encuentran a temperaturas diferentes (Sateesha et al., 2018).

Los intercambiadores de calor se utilizan en diferentes industrias tales como la petroquímica, biotecnológica, energética, de refrigeración y alimenticia, entre otras, mientras que en la actualidad existe una demanda creciente en cuanto al diseño y construcción de intercambiadores de calor más eficientes desde el punto de vista térmico.

El desarrollo de la industria global ha conllevado al diseño de nuevos intercambiadores compactos para incrementar la tasa de transferencia de calor, reducir el tamaño de los intercambiadores de calor y aumentar la efectividad del proceso térmico que se lleva a cabo en estos equipos (Borse \& Bute, 2018). Entre los tipos de intercambiadores de calor que han surgido como alternativa a los diseños típicos se encuentra el de serpentín, el cual consiste básicamente en un serpentín helicoidal fabricado de una tubería metálica, la cual se encuentra colocada en la porción anular conformada por dos cilindros concéntricos (Fig. 1).

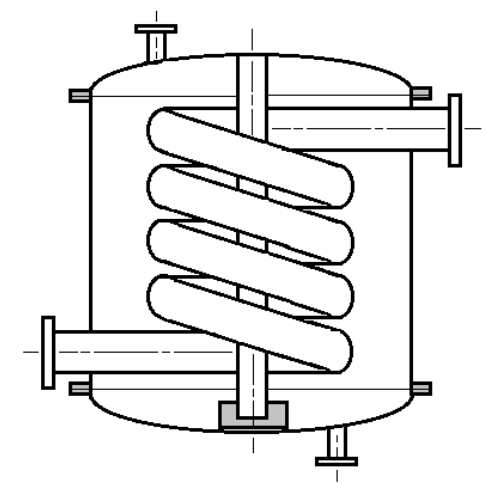

Figura 1. Intercambiador de calor de serpentín Fuente: Elaboración propia

En estos equipos los fluidos fluyen por dentro del serpentín y el ánulo, en donde la transferencia de calor ocurre a través de la pared del serpentín. Las dimensiones de ambos cilindros están determinadas por la 


\section{A. Pérez Sánchez et al.}

velocidad del fluido que circula por el ánulo necesaria para cumplir con las necesidades de transferencia de calor del sistema (Patil et al., 1982).

El uso de intercambiadores de calor de serpentín (ICS) para diferentes aplicaciones y procesos ha sido estudiado por diferentes investigadores (Prabhanjan et al., 2002) (Jayakumar et al., 2008) (Kharat et al., 2009) (San et al., 2012) (Ahire et al., 2014) (Ankann \& Reddy, 2014) (Andhare et al., 2014) (Lazova et al., 2017). En estos equipos, la fuerza centrífuga que tiene lugar debido a la curvatura del tubo trae consigo el desarrollo de un flujo secundario. De esta manera, la curvatura del serpentín gobierna la fuerza centrífuga, mientras que el paso o ángulo de la hélice influye en la torsión a la que está sujeta el fluido. A causa de la curvatura, el fluido que circula por el interior del serpentín se mueve a una mayor velocidad en comparación con el fluido que fluye por fuera del serpentín. Esta diferencia de velocidad ocasiona la aparición de un flujo secundario el cual aumenta la tasa de transferencia de calor (Borse \& Bute, 2018). Los ICS se emplean fundamentalmente cuando (Patil et al., 1982):

- Existe limitación de espacio.

- Existen condiciones de flujo laminar o bajos caudales másicos, en donde un intercambiador de calor de tubo y coraza sería incosteable debido a los bajos coeficientes de transferencia de calor resultantes.

- La caída de presión de un fluido está limitada a cierto valor de diseño (por ejemplo, debido al flujo del fluido a través de otros equipos de proceso)

En el presente trabajo se lleva a cabo el diseño de un ICS para realizar el enfriamiento de una corriente de acetona líquida. También se determina la caída de presión de ambos fluidos y se comparan los valores obtenidos con el límite máximo establecido por el proceso para este parámetro. Por último, se determina la influencia del incremento de dos variables iniciales (caudal másico de alimentación de la acetona y temperatura de entrada de la acetona) sobre cuatro parámetros de diseño del ICS, los cuales son: coeficiente global de transferencia de calor; área de transferencia de calor, número de vueltas del serpentín y altura del intercambiador.

\section{MATERIALES Y MÉTODOS}

\subsection{Descripción del problema}

Se necesita diseñar un ICS para llevar a cabo el enfriamiento de $300 \mathrm{~kg} / \mathrm{h}$ de una corriente de acetona líquida, desde $70{ }^{\circ} \mathrm{C}$ hasta $30{ }^{\circ} \mathrm{C}$, empleando agua fría a $2{ }^{\circ} \mathrm{C}$ como medio de enfriamiento. Para ello se dispone de los siguientes datos para el serpentín y los cilindros tanto interior como exterior (Fig. 2):

- Diámetro exterior del cilindro interior (B): 0,32 m.

- Diámetro interior del cilindro exterior $\left(\mathrm{D}_{\mathrm{i}}\right): 0,48 \mathrm{~m}$.

- Diámetro interior del serpentín $\left(\mathrm{d}_{\mathrm{i}}\right): 0,027 \mathrm{~m}$.

- Diámetro exterior del serpentín $\left(\mathrm{d}_{\mathrm{e}}\right): 0,032 \mathrm{~m}$.

- Diámetro promedio de la hélice $\left(\mathrm{D}_{\mathrm{h}}\right): 0,400 \mathrm{~m}$.

La temperatura de salida del agua de enfriamiento no deberá exceder $\operatorname{los} 7{ }^{\circ} \mathrm{C}$, y se escoge un factor de ensuciamiento para el agua y la acetona de $\mathrm{R}_{\mathrm{H} 2 \mathrm{O}}=0,0002$ y $\mathrm{R}_{\text {acet }}=0,0004 \mathrm{hm} 2^{\circ} \mathrm{C} / \mathrm{kcal}$, respectivamente (Cao, 2010). Se ubicará el agua de enfriamiento por el interior del serpentín, mientras que la acetona circulará por el ánulo o coraza, y los fluidos circulan en el interior del intercambiador en un arreglo en 


\section{A. Pérez Sánchez et al.}

paralelo. El material del serpentín será acero inoxidable 304, y tendrá una conductividad térmica $\left(\mathrm{k}_{\mathrm{t}}\right)$ de 14 $\mathrm{kcal} / \mathrm{h} . \mathrm{m} .{ }^{\circ} \mathrm{C}$ (Perry \& Green, 2008). La caída de presión del fluido que circula por la coraza y del fluido que circula por el interior del serpentín no deberá exceder los $0,5 \mathrm{~Pa}$ y $20 \mathrm{kPa}$, respectivamente.

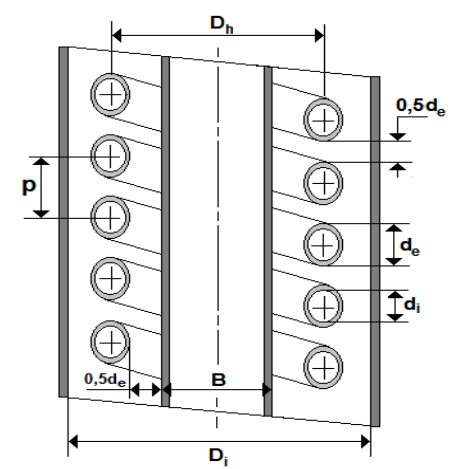

Figura 2. Vista esquemática de corte de un intercambiador de calor de serpentín Fuente: Elaboración propia

\subsection{Temperatura promedio de cada fluido}

La temperatura promedio de cada fluido se determinó mediante las ecuaciones (1) y (2):

Acetona:

$$
\bar{T}=\frac{T_{e}+T_{s}}{2}=\frac{70+30}{2}=50^{\circ} \mathrm{C}
$$

Agua de enfriamiento:

$$
\bar{t}=\frac{t_{e}+t_{s}}{2}=\frac{2+7}{2}=4,5^{\circ} \mathrm{C}
$$

\subsection{Propiedades termofísicas de los fluidos}

La Tabla 1 muestra los valores de las diferentes propiedades termofísicas de ambos fluidos tomadas de (Perry \& Green, 2008), las cuales fueron determinadas a la temperatura promedio de cada corriente calculadas en el acápite anterior.

Tabla 1. Propiedades termofísicas de ambos fluidos de trabajo

\begin{tabular}{cccc}
\hline Propiedad & Acetona & Agua & Unidades \\
\hline Calor específico $(\mathrm{Cp})$ & 0,540 & 1,007 & $\mathrm{kcal} / \mathrm{kg} .{ }^{\circ} \mathrm{C}$ \\
Densidad $(\rho)$ & 757,3 & 1003,2 & $\mathrm{~kg} / \mathrm{m} 3$ \\
Viscosidad $(\mu)$ & 0,889 & 5,415 & $\mathrm{~kg} / \mathrm{m} . \mathrm{h}$ \\
Conductividad térmica $(\mathrm{k})$ & 0,1288 & 0,4942 & $\mathrm{kcal} / \mathrm{h} . \mathrm{m} .{ }^{\circ} \mathrm{C}$ \\
\hline
\end{tabular}

Fuente: Elaboración propia

2.4 Metodología de diseño del intercambiador de calor 


\section{A. Pérez Sánchez et al.}

El diseño del intercambiador de calor de serpentín se efectuó mediante el empleo de correlaciones y ecuaciones publicadas fundamentalmente en (Patil et al. 1982) (Kern, 1999) (Perry \& Green, 2008). De esta manera, primeramente, se determina el calor intercambiado en el sistema $(\mathrm{Q})$ y el caudal másico requerido de agua de enfriamiento, a través de las ecuaciones (3) y (4), respectivamente.

$$
\begin{gathered}
Q=m_{\text {acet }} \cdot C p_{\text {acet }} \cdot\left(T_{e}+T_{s}\right) \\
m_{\text {agua }}=\frac{Q}{C p_{\text {agua }} \cdot\left(t_{s}-t_{e}\right)}
\end{gathered}
$$

A continuación, se determinan el paso (p) [ecuación (5)], el diámetro interior de la hélice ( $\left.\mathrm{Di}_{\mathrm{h}}\right)$ [ec. (6)] y el diámetro exterior de la hélice $\left(\mathrm{De}_{\mathrm{h}}\right)$ [ec. (7)]:

$$
\begin{gathered}
p=1,5 \cdot d_{e} \\
D i_{h}=B+d_{e} \\
D e_{h}=B+2 \cdot d_{e}+d_{e}
\end{gathered}
$$

Luego se calcula la longitud necesaria del serpentín ( $\mathrm{L}_{\text {serp }}$ ) [ec. (8)]; el volumen ocupado por el serpentín $\left(\mathrm{V}_{\text {serp }}\right)$ [ec.(9)]; el volumen del ánulo $\left(\mathrm{V}_{\mathrm{a}}\right)$ [ec. (10)]; el volumen disponible para el flujo en el ánulo ( $\left.\mathrm{V}_{\text {flujo }}\right)$ [ec. (11)]; el diámetro equivalente de la coraza $\left(\mathrm{D}_{\text {eq }}\right)$ [ec. (12)]; la velocidad másica del fluido que circula por la coraza (acetona) $\left(\mathrm{G}_{\text {acet }}\right)$ [ec. (13)]; el número de Reynolds de la acetona $\left(\mathrm{Re}_{\text {acet }}\right)$ [ec. (14)]; el número de Prandtl de la acetona $\left(\mathrm{Pr}_{\text {acet }}\right.$ ) [ec. (15)]; y finalmente el coeficiente pelicular de transferencia de calor de la acetona $\left(\mathrm{h}_{0}\right)$ [ec. $\left.(16)\right]$

$$
\begin{gathered}
L_{\text {serp }}=N \cdot \sqrt{\left(2 \cdot \pi \cdot \frac{D_{h}}{2}\right)+p^{2}} \\
V_{\text {serp }}=\left(\frac{\pi}{4}\right) \cdot d_{e}^{2} \cdot L_{\text {serp }} \\
V_{a}=\left(\frac{\pi}{4}\right) \cdot\left(D_{i}^{2}-B^{2}\right) \cdot p \\
V_{\text {flujo }}=V_{a}-V_{\text {serp }} \\
D_{\text {eq }}=\frac{4 \cdot V_{\text {flujo }}}{\pi \cdot d_{e} \cdot L_{\text {serp }}} \\
=\frac{m_{\text {acet }}}{\left[\left(\frac{\pi}{4}\right) \cdot\left(\left(D_{i}^{2}-B^{2}\right)-\left(D e_{h}^{2}-D i_{h}^{2}\right)\right)\right]} \\
\operatorname{Re}_{a c e t}=\frac{D_{e q} \cdot G_{\text {acet }}}{\mu_{\text {acet }}} \\
\operatorname{Pr}_{a c e t}=\frac{C p_{\text {acet }} \cdot \mu_{\text {acet }}}{k_{\text {acet }}} \\
h_{0}=0,6 \cdot \frac{k}{D_{e q}} \cdot \operatorname{Re}_{\text {acet }}^{0,5} \cdot \operatorname{Pr}_{a c e t}^{0,31}
\end{gathered}
$$

Válida para $50<\operatorname{Re}<10000$ 
Posteriormente se computa el área de sección transversal del serpentín $\left(\mathrm{A}_{\text {serp }}\right)$ [ec. (17)]; el caudal volumétrico del agua ( $\mathrm{q}_{\text {agua }}$ [ [ec. (18)]; la velocidad del agua ( $\mathrm{v}_{\text {agua }}$ [ [ec. (19)]; el número de Reynolds del agua $\left(\operatorname{Re}_{\text {agua }}\right)$ [ec. (20)]; el número de Prandtl del agua $\left(\operatorname{Pr}_{\text {agua }}\right)$ [ec. (21)]; el coeficiente pelicular de transferencia de calor del agua basado en el diámetro interior del serpentín para el tubo recto $\left(\mathrm{h}_{\mathrm{i}}\right)$ [ec. (22)]; el coeficiente pelicular de transferencia de calor del agua corregido para el tubo enroscado ( $\mathrm{h}_{\text {ic }}$ ) [ec. (23)]; y por último el coeficiente pelicular de transferencia de calor basado en el diámetro exterior del serpentín $\left(\mathrm{h}_{\mathrm{i} 0}\right)$ [ec. (24)].

$$
\begin{gathered}
A_{\text {serp }}=\frac{\pi \cdot d_{i}^{2}}{4} \\
q_{\text {agua }}=\frac{m_{\text {agua }}}{\rho_{\text {agua }}} \\
v_{\text {agua }}=\frac{q_{\text {agua }}}{A_{\text {serp }}} \\
\operatorname{Re}_{\text {agua }}=\frac{d_{i} \cdot v_{\text {agua }} \cdot \rho_{\text {agua }}}{\mu_{\text {agua }}} \\
\operatorname{Pr}_{\text {agua }}=\frac{C p_{\text {agua }} \cdot \mu_{\text {agua }}}{k_{\text {agua }}} \\
h_{i}=\frac{k_{\text {agua }}}{d_{i}} \cdot 0,023 \cdot \operatorname{Re}_{\text {agua }}^{0,8} \cdot \operatorname{Pr}_{\text {agua }}^{0,33} \cdot\left(\frac{\mu_{\text {agua }}}{\mu_{P}}\right)^{0,14}
\end{gathered}
$$

Se asume que $\left(\mu_{\text {agua }} / \mu_{\text {agua(P) }}\right)^{0.14}$ es igual a $1(\mathrm{Cao}, 2010)$

$$
\begin{gathered}
h_{i c}=h_{i} \cdot\left[1+3,5 \cdot\left(\frac{d_{i}}{D_{h}}\right)\right] \\
h_{i 0}=h_{i c} \cdot\left(\frac{d_{i}}{d_{e}}\right)
\end{gathered}
$$

Finalmente se determina el espesor del tubo serpentín ( $e_{t}$ ); el coeficiente global de transferencia de calor (U); la Media Logarítmica de la Diferencia de Temperatura (MLDT) para un arreglo de flujo en paralelo; la diferencia de temperatura corregida $(\Delta \mathrm{t})$; el área de transferencia de calor requerida (A); y el número teórico de vueltas del serpentín (N), mediante las ecuaciones (25) (26) (27) (28) (29) y (30), respectivamente.

$$
\begin{gathered}
e_{t}=\frac{d_{e}-d_{i}}{2} \\
U=\frac{1}{\frac{1}{h_{0}}+\frac{1}{h_{i 0}}+\frac{x_{t}}{k_{t}}+R_{\text {agua }}+R_{\text {ace }}} \\
M L D T=\frac{\left(T_{e}-t_{e}\right)-\left(T_{s}-t_{s}\right)}{\ln \frac{\left(T_{e}-t_{e}\right)}{\left(T_{s}-t_{s}\right)}} \\
\Delta t=M L D T \cdot F_{t}
\end{gathered}
$$


Donde $\mathrm{Ft}=0,99(\mathrm{Cao}, 2010)($ Kern, 1999)

$$
N=\frac{A=\frac{Q}{U \cdot \Delta t}}{\left[\pi \cdot d_{e} \cdot\left(\frac{L_{\text {serp }}}{N}\right)\right]}
$$

El número real de vueltas del serpentín (n) se determinará simplemente redondeando el valor de $\mathrm{N}$ obtenido mediante la ecuación (30) hacia el próximo número entero superior, mientras que la altura de cilindro necesaria para acomodar el número real de vueltas del serpentín $(\mathrm{H})$ se determina mediante la siguiente ecuación:

$$
H=n \cdot p+d_{e}
$$

\subsection{Caída de presión}

La caída de presión se determinará mediante el empleo de correlaciones publicadas en (Brauer, 1964) (Andrzejczyk \& Muszyński, 2016). De esta manera, la velocidad de la acetona ( $\mathrm{v}_{\text {acet }}$ ); la velocidad del agua ( $\mathrm{v}^{\prime}$ agua $)$; el factor $\mathrm{E}$; el factor de fricción del fluido que circula por el interior del serpentín ( $\mathrm{f}_{\text {agua }}$ ) y el coeficiente de arrastre $\left(C_{A}\right)$ se determinan mediante las ecuaciones (32) (33) (34) (35) y (36).

$$
\begin{gathered}
\frac{G_{\text {acet }}}{\rho_{\text {acet }}} \\
v_{\text {acet }} \\
v_{\text {agua }}^{\prime}=\frac{v_{\text {agua }}}{3600} \\
E=D_{h} \cdot\left[1+\left(\frac{p}{\pi \cdot D_{h}}\right)^{2}\right] \\
f_{\text {agua }}=\left[\frac{0,3164}{\operatorname{Re}_{\text {agua }}^{0,25}}+0,03 \cdot\left(\frac{d_{i}}{E}\right)^{0,5}\right] \cdot\left(\frac{\mu_{\text {agua }(p)}}{\mu_{\text {agua }}}\right)^{0,27} \\
C_{A}=\frac{0,3164}{\operatorname{Re}_{\text {acet }}^{0,25} \cdot\left[1+0,095 \cdot\left(\frac{d_{e}}{D_{h}}\right)^{0,5} \cdot \operatorname{Re}_{\text {acet }}^{0,25}\right]}
\end{gathered}
$$

Por último, la caída de presión del fluido que circula por la coraza (acetona) se determina mediante la ecuación (37), mientras que la caída de presión del fluido que circula por el serpentín (agua de enfriamiento) se calcula a partir de la ecuación (38).

$$
\begin{gathered}
\Delta P_{\text {acet }}=C_{A} \cdot \frac{H}{D_{\text {eq }}} \cdot \frac{v_{\text {acet }}^{2} \cdot \rho_{\text {acet }}}{2} \\
\Delta P_{\text {agua }}=f_{\text {agua }} \cdot \frac{L_{\text {serp }}}{d_{i}} \cdot \frac{v_{\text {agua }}^{2} \cdot \rho_{\text {acet }}}{2}
\end{gathered}
$$




\section{A. Pérez Sánchez et al.}

2.6 Determinación de la influencia del caudal de alimentación y la temperatura de entrada de la acetona sobre cuatro parámetros de diseño del intercambiador de calor de serpentín

Se determinó la influencia que presenta el incremento de tanto el caudal de alimentación de la acetona $\left(\mathrm{m}_{\text {acet }}\right)$ como su temperatura de entrada $\left(\mathrm{T}_{\mathrm{e}}\right)$ sobre cuatro parámetros de diseño del ICS, a saber: coeficiente global de transferencia de calor (U), área de transferencia de calor (A), número real de vueltas del serpentín (n), y altura del cilindro (H). A tal fin se variará el valor de $m_{\text {acet }}$ entre $260-350 \mathrm{~kg} / \mathrm{h}$, y el valor de Te entre $66-75{ }^{\circ} \mathrm{C}$, y se graficarán los resultados obtenidos para cada uno de los cuatro parámetros considerados tomando en cuenta esta variación. Lo anterior se realiza con el objetivo de predecir qué valores tendrán los principales parámetros de diseño del intercambiador de calor de serpentín ante cualquier futura variación de tanto el caudal de alimentación como la temperatura de entrada de la acetona. Por último se obtendrá la ecuación de ajuste para cada uno de los gráficos obtenidos.

\section{RESULTADOS Y DISCUSIÓN}

\subsection{Diseño del intercambiador de calor de serpentín}

La siguiente tabla (Tabla 2) muestra los resultados obtenidos durante el diseño del intercambiador de calor de serpentín empleando la metodología de cálculo plantada con anterioridad.

Tabla 2. Resultados alcanzados durante el diseño del intercambiador de calor de serpentín.

\begin{tabular}{|c|c|c|c|}
\hline Variable & Valor & Unidad & Ecuación \\
\hline Calor intercambiado $(\mathrm{Q})$ & 6479 & $\mathrm{kcal} / \mathrm{h}$ & (3) \\
\hline Caudal másico de agua ( $\mathrm{m}_{\text {agua }}$ ) & 1286,7 & $\mathrm{~kg} / \mathrm{h}$ & (4) \\
\hline Paso (p) & 0,048 & $\mathrm{~m}$ & (5) \\
\hline Diámetro interior de la hélice $\left(\mathrm{D}_{\mathrm{ih}}\right)$ & 0,352 & $\mathrm{~m}$ & (6) \\
\hline Diámetro exterior de la hélice $\left(\mathrm{D}_{\mathrm{eh}}\right)$ & 0,416 & $\mathrm{~m}$ & (7) \\
\hline Longitud necesaria del serpentín ( $\mathrm{L}_{\text {serp }}$ ) & $1,257 \mathrm{~N}$ & $\mathrm{~m}$ & (8) \\
\hline Volumen ocupado por el serpentín $\left(\mathrm{V}_{\text {serp }}\right)$ & $0,0010 \mathrm{~N}$ & m3 & (9) \\
\hline Volumen del ánulo (Va) & $0,0048 \mathrm{~N}$ & $\mathrm{~m} 3$ & (10) \\
\hline Volumen disponible para el flujo en el ánulo $\left(\mathrm{V}_{\text {flujo }}\right)$ & $0,0038 \mathrm{~N}$ & m3 & (11) \\
\hline Diámetro equivalente de la coraza $\left(\mathrm{D}_{\mathrm{eq}}\right)$ & 0,1208 & $\mathrm{~m}$ & (12) \\
\hline Velocidad másica de la acetona $\left(\mathrm{G}_{\mathrm{acet}}\right)$ & 4847 & $\mathrm{~kg} / \mathrm{m}^{2} . \mathrm{h}$ & (13) \\
\hline Número de Reynolds de la acetona $\left(\operatorname{Re}_{\text {acet }}\right)$ & 659 & & (14) \\
\hline Número de Prandtl de la acetona $\left(\operatorname{Pr}_{\text {acet }}\right)$ & 3,72 & & $(15)$ \\
\hline Coeficiente de transferencia de calor de la acetona $\left(\mathrm{h}_{0}\right)$ & 24,7 & $\mathrm{kcal} / \mathrm{h} \cdot \mathrm{m}^{2} \cdot{ }^{\circ} \mathrm{C}$ & (16) \\
\hline Área de sección transversal del serpentín ( $\left.A_{\text {serp }}\right)$ & 0,00057 & $\mathrm{~m} 2$ & (17) \\
\hline Caudal volumétrico del agua (qagua) & 1,283 & $\mathrm{~m}^{3} / \mathrm{h}$ & (18) \\
\hline Velocidad del agua ( $\mathrm{V}_{\text {agua }}$ ) & 2241,20 & $\mathrm{~m} / \mathrm{h}$ & (19) \\
\hline Número de Reynolds del agua $\left(\operatorname{Re}_{\text {agua }}\right)$ & 11211 & & (20) \\
\hline Número de Prandtl del agua $\left(\operatorname{Pr}_{\text {agua }}\right)$ & 11,03 & & (21) \\
\hline $\begin{array}{l}\text { Coeficiente de transferencia de calor del agua basado en el } \\
\text { diámetro interior del serpentín para el tubo recto }\left(\mathrm{h}_{\mathrm{i}}\right)\end{array}$ & 1614,73 & $\mathrm{kcal} / \mathrm{h} \cdot \mathrm{m}^{2} \cdot{ }^{\circ} \mathrm{C}$ & (22) \\
\hline Coeficiente de transferencia de calor del agua corregido para el & 1996,21 & $\mathrm{kcal} / \mathrm{h} \cdot \mathrm{m}^{2} \cdot{ }^{\circ} \mathrm{C}$ & (23) \\
\hline
\end{tabular}




\section{A. Pérez Sánchez et al.}

tubo enroscado $\left(\mathrm{h}_{\mathrm{ic}}\right)$

Coeficiente de transferencia de calor basado en el diámetro

exterior del serpentín $\left(\mathrm{h}_{\mathrm{i} 0}\right)$

Espesor del tubo serpentín $\left(\mathrm{x}_{\mathrm{t}}\right)$

$1684,30 \quad \mathrm{kcal} / \mathrm{h} . \mathrm{m}^{2}{ }^{\circ} \mathrm{C}$

Coeficiente global de transferencia de calor (U)

0,0025

$\mathrm{m}$

Media Logarítmica de la Diferencia de Temperatura (MLDT)

23,88

41,51

$\mathrm{kcal} / \mathrm{h} \cdot \mathrm{m}^{2} \cdot{ }^{\circ} \mathrm{C}$

Diferencia de temperatura corregida $(\Delta \mathrm{t})$

41,10

${ }^{\circ} \mathrm{C}$

Área de transferencia de calor requerida (A)

6,60

${ }^{\circ} \mathrm{C}$

Número teórico de vueltas del serpentín (N)

52,26

Número real de vueltas del serpentín (n)

Altura de cilindro necesaria para acomodar el número real de

2,58 vueltas del serpentín $(\mathrm{H})$

Fuente: Elaboración propia

Tomando en cuenta los resultados de la Tabla 2, se puede concluir que el ICS tendrá un coeficiente global de transferencia de calor de $23,88 \mathrm{kcal} / \mathrm{h} \cdot \mathrm{m}^{2}{ }^{\circ} \mathrm{C}$, un área de transferencia de calor de $6,60 \mathrm{~m}^{2}$, un número real de vueltas del serpentín igual a 53 , y una altura total de $2,58 \mathrm{~m}$.

\subsection{Caída de presión}

La Tabla 3 muestra los resultados obtenidos con respecto a la caída de presión calculada para ambas corrientes.

Tabla 3. Resultados de la caída de presión para ambos fluidos

\begin{tabular}{lccc}
\hline Variable & Valor & Unidad & Ecuación \\
\hline Velocidad de la acetona $\left(\mathrm{v}_{\text {acet }}\right)$ & 0,002 & $\mathrm{~m} / \mathrm{s}$ & $(32)$ \\
Velocidad del agua $\left(\mathrm{v}^{\prime} \mathrm{a}_{\text {gua }}\right)$ & 0,595 & $\mathrm{~m} / \mathrm{s}$ & $(33)$ \\
Factor E & 0,401 & $\mathrm{~m}$ & $(34)$ \\
Factor de fricción del agua $\left(\mathrm{f}_{\text {agua }}\right)$ & 0,039 & & $(35)$ \\
Coeficiente de arrastre $\left(\mathrm{C}_{\mathrm{A}}\right)$ & 0,070 & $\mathrm{~Pa}$ & $(36)$ \\
Caída de presión de la acetona $\left(\Delta \mathrm{P}_{\text {acet }}\right)$ & 0,2 & $\mathrm{~Pa}$ & $(38)$ \\
Caída de presión del agua $\left(\Delta \mathrm{P}_{\text {agua }}\right)$ & 16188 & & \\
\hline Fuente: Elaboración propia & & &
\end{tabular}

Considerando los resultados obtenidos en las ecuaciones (37) y (38) de la Tabla 3 se concluye que tanto la corriente de acetona como la de agua de enfriamiento presentan resultados caída de presión inferiores al límite máximo permisible establecido por el proceso $(0,5 \mathrm{~Pa}$ para la acetona y $18 \mathrm{kPa}$ para el agua de enfriamiento).

3.3 Influencia del caudal de alimentación y temperatura de entrada de la acetona sobre 4 parámetros de diseño del ICS

Las figuras 3 y 4 muestran los gráficos obtenidos durante la evaluación de la influencia del incremento del caudal de alimentación (macet) y la temperatura de entrada (Te) de la acetona, respectivamente, sobre cuatro parámetros de diseño del ICS: 1) coeficiente global de transferencia de calor (U), 2) área de transferencia de calor (A), 3) número real de vueltas del serpentín (n) y 4) altura del equipo (H). 


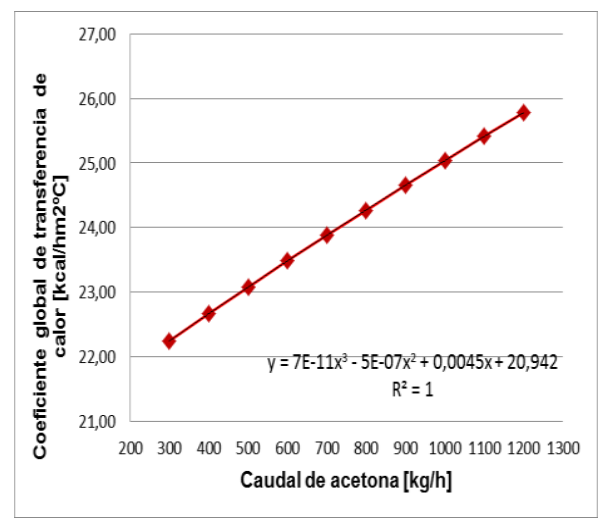

a)

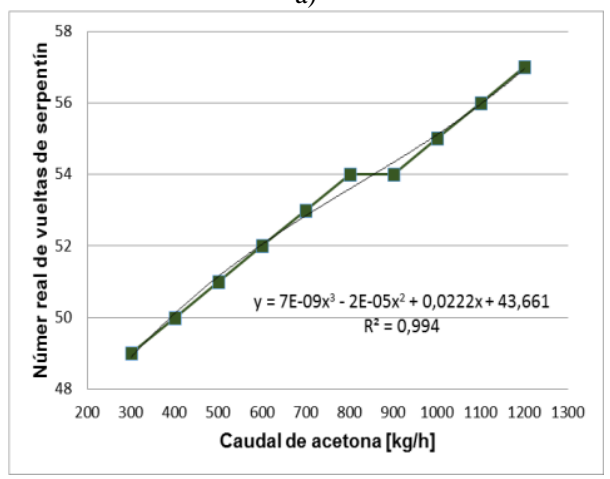

c)

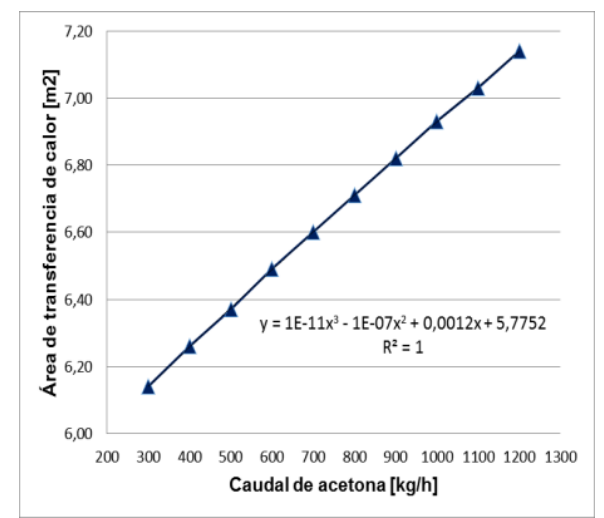

b)

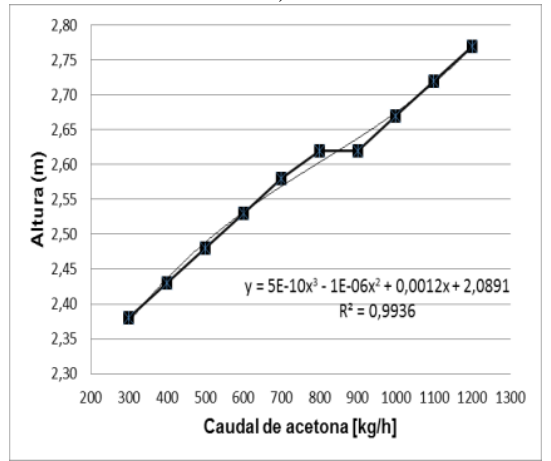

d)

Figura 3. Influencia del incremento del caudal de alimentación de la acetona sobre 4 parámetros de diseño el intercambiador de calor de serpentín. (a) Coeficiente global de transferencia de calor. (b) Área de transferencia de calor. (c) Número real de vueltas del serpentín. (d) Altura del equipo.

Fuente: Elaboración propia.

El incremento del caudal de alimentación de la acetona $\left(\mathrm{m}_{\text {acet }}\right)$ origina un aumento del número de Reynolds de la acetona, lo cual produce, a su vez, un incremento del coeficiente pelicular de transferencia de calor de la acetona $\left(h_{0}\right)$ de acuerdo con la ecuación (16). El incremento de $h_{0}$ provoca como resultado un aumento del valor del coeficiente global de transferencia de calor (U), como se plantea en la ecuación (26). El incremento de $m_{\text {acet }}$ también origina un incremento del calor intercambiado (Q) según la ecuación (3), lo cual influye de forma directa en el aumento del valor del área de transferencia de calor de acuerdo con la ecuación (29). Mientras mayor sea el caudal de alimentación de la acetona, mayor será el área de transferencia de calor necesaria para cumplir con la demanda térmica de este sistema de transferencia de calor. De esta manera, a mayor área de transferencia de calor que se necesite, mayor será el número real de vueltas de serpentín requerido, y con ello, mayor será también la altura que tendrá el ICS, según la ecuación (31).

Por otro lado, el incremento de $\mathrm{m}_{\text {acet }}$ también involucra el aumento del caudal de agua de enfriamiento (magua) requerido para hacerle frente al incremento del calor intercambiado (Q), lo cual influye, a su vez, en el aumento de número de Reynolds del agua, en el incremento del coeficiente pelicular de transferencia de calor del agua $\left(\mathrm{h}_{\mathrm{i} 0}\right) \mathrm{y}$, por consiguiente, en el aumento del coeficiente global de transferencia de calor (U).

De esta manera se concluye que el incremento de $\mathrm{m}_{\text {acet }}$ causa el aumento de los 4 parámetros de diseño seleccionados. 


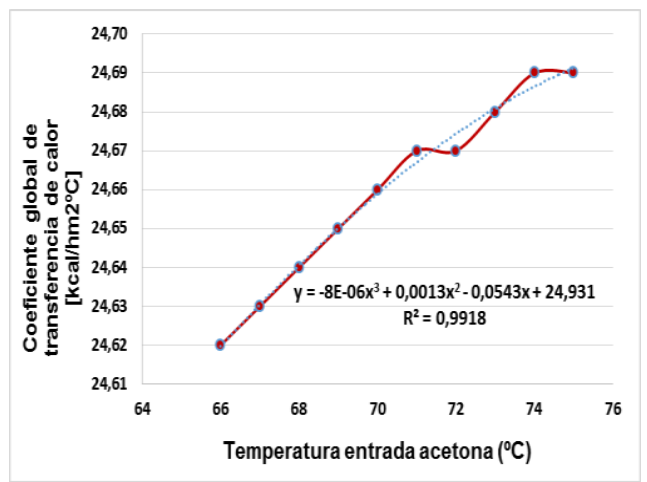

a)

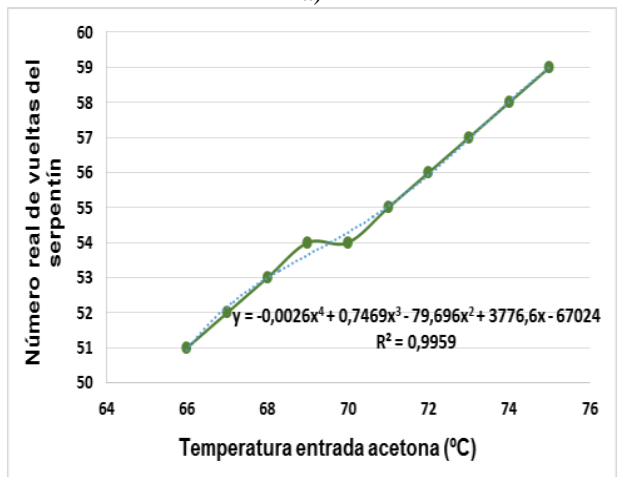

c)

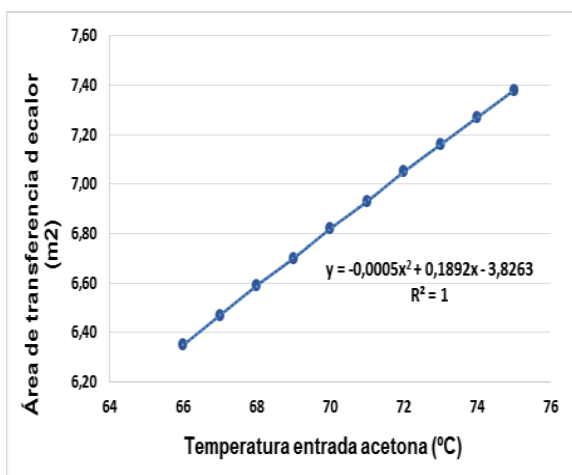

b)

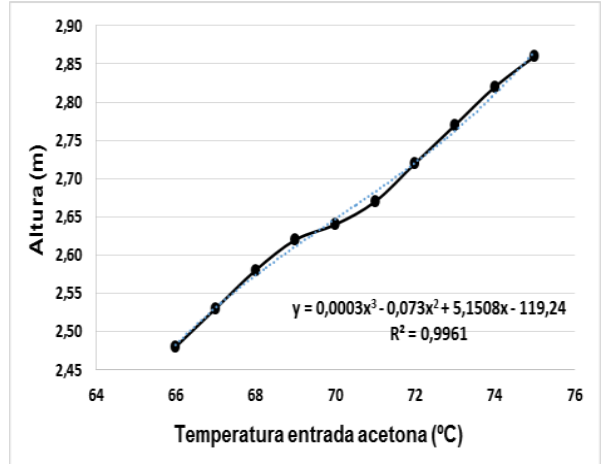

d)

Figura 4. Influencia del incremento de la temperatura de entrada de la acetona sobre 4 parámetros de diseño el intercambiador de calor de serpentín. (a) Coeficiente global de transferencia de calor. (b) Área de transferencia de calor. (c) Número de vueltas del serpentín. (d) Altura del equipo.

Fuente: Elaboración propia.

Un incremento de la temperatura de entrada de la acetona $\left(\mathrm{T}_{\mathrm{e}}\right)$ provoca el incremento de tanto el calor intercambiado $(\mathrm{Q})$ como del caudal de agua $\left(\mathrm{m}_{\text {agua }}\right)$ necesario para enfriar la acetona hasta la temperatura deseada $\left(30^{\circ} \mathrm{C}\right)$. El aumento de magua provoca el incremento del número de Reynolds del agua, con el consiguiente aumento del coeficiente pelicular de transferencia de calor del agua $\left(\mathrm{h}_{\mathrm{i} 0}\right) \mathrm{y}$, por tanto, el incremento del coeficiente global de transferencia de calor $(\mathrm{U})$. Por otro lado, a mayor valor de $\mathrm{Q}$, mayor área de transferencia de calor (A) será necesaria, por lo que un incremento de A ocasiona un aumento del número real de vueltas del serpentín, y por tanto, un aumento de la altura del equipo. Vale destacar que A es directamente proporcional a $\mathrm{Q}$ e inversamente proporcional a $\mathrm{U}$, sin embargo la mayor tasa de incremento obtenida para $A$ con respecto a la tasa de incremento alcanzada para $U$, condiciona que se obtenga un valor de A creciente a medida que aumenta Te. También ocurre que el coeficiente pelicular de la acetona $\left(\mathrm{h}_{0}\right)$ se mantiene constante en un valor de $24,7 \mathrm{kcal} / \mathrm{h} . \mathrm{m}^{2}{ }^{\circ} \mathrm{C}$ durante todo el rango de temperatura aplicado en este estudio $\left(66-75^{\circ} \mathrm{C}\right)$, lo cual se debe a que la misma tasa de incremento que se observa en el número de Reynolds de la acetona ( $\left.\mathrm{Re}_{\mathrm{acet}}\right)$ se observa en el número de Prandtl de la acetona $\left(\mathrm{Pr}_{\text {acet }}\right)$, pero de forma inversa, es decir, mientras que $\mathrm{Re}_{\text {acet }}$ aumenta, $\mathrm{Pr}_{\text {acet }}$ disminuye, ocurriendo ambos procesos a la misma tasa, lo cual origina que se obtenga un valor constante de h0 en todo el intervalo de temperatura aplicado.

Por último, la diferencia de temperatura corregida $(\Delta t)$, la cual es inversamente proporcional a $\mathrm{A}$, se incrementa a medida que Te aumenta, aunque según lo observado, este aumento es tan poco significativo (aumenta desde $39,30^{\circ} \mathrm{C}$ a una $\mathrm{T}_{\mathrm{e}}=65^{\circ} \mathrm{C}$, hasta $42,86^{\circ} \mathrm{C}$ a una $\mathrm{Te}=75^{\circ} \mathrm{C}$ ) que apenas tiene influencia en el incremento que experimenta $\mathrm{A}$ a medida que Te aumenta. 


\section{A. Pérez Sánchez et al.}

Considerando los resultados obtenidos se concluye que los cuatro parámetros evaluados (U, A, n y H) se incrementan a medida que Te aumenta.

\section{CONCLUSIONES}

El intercambiador de calor de serpentín diseñado tendrá un coeficiente global de transferencia de calor (U) de $23,88 \mathrm{kcal} / \mathrm{h} . \mathrm{m}^{2}{ }^{\circ} \mathrm{C}$; un área de transferencia de calor (A) de $6,60 \mathrm{~m}^{2}$; un número real de vueltas de serpentín (n) de 53 y una altura $(\mathrm{H})$ de $2,58 \mathrm{~m}$.

La acetona y el agua de enfriamiento presentan valores de caída de presión de 0,2 Pa y 16,19 kPa, respectivamente, las cuales son inferiores al límite máximo establecido para el proceso para ambas corrientes.

El incremento de tanto el caudal de alimentación como la temperatura de entrada de la acetona aumenta los valores de los parámetros $\mathrm{U}, \mathrm{A}, \mathrm{n}$ y $\mathrm{H}$.

\section{REFERENCIAS}

Ahire, S., Shelke, P., Shinde, B., and Totala, N. (2014). Fabrication and Analysis of Counter Flow Helical Coil Heat Exchanger. International Journal of Engineering Trends and Technology, 15 (5), 229-240.

Andhare, A., Kriplani, V. M., and Modak, J. P. (2014). Thermal Analysis of a Helical Coil Heat Exchanger. International Journal of Innovative Research in Advanced Engineering, 1 (12), 135-143.

Andrzejczyk, R., and Muszyński, T. (2016). Performance analyses of helical coil heat exchangers. The effect of external coil surface modification on heat exchanger effectiveness. Archives of Thermodynamics, 37 (4), 137-159.

Ankann, B. C., and Reddy, B. S. (2014). Performance analysis of fabricated helical coil heat exchanger. International Journal of Engineering Research, 3 (Special 1), 33-39.

Borse, D., and Bute, J. V. (2018). A Review on Helical Coil Heat Exchanger. International Journal for Research in Applied Science \& Engineering Technology, 6 (2), 492-497.

Brauer, H. (1964). Strömungswiderstand und Wärmeübergang bei quer angeströmten Wärmeaustauschern mit kreuzgitterförmig angeordneten glatten und berippten Rohren. Chemie Ing. Tech., 36, 247-260.

Cao, E. (2010). Heat transfer in process engineering. New York, USA: McGraw-Hill.

Jayakumar, J. S., Mahajani, S. M., Mandal, J. C., Vijayan, P. K., and Bhoi, R. (2008). Experimental and CFD estimation of heat transfer in helically coiled heat exchangers. Chemical Engineering Research and Design, 86, 221-232.

Kern, D. Q. (1999). Procesos de Transferencia de Calor. México D.F, México: Compañía Editorial Continental S.A de C.V.

Kharat, R., Bhardwaj, N., and Jha, R. S. (2009). Development of heat transfer coefficient correlation for concentric helical coil heat exchanger. International Journal of Thermal Sciences, 48, 2300-2308. 


\section{A. Pérez Sánchez et al.}

Lazova, M., Kaya, A., Billiet, M., Lecompte, S., Manolakos, D., and Paepe, M. D. (2017). Experimental Assessment of a Helical Coil Heat Exchanger Operating at Subcritical and Supercritical Conditions in a Small-Scale Solar Organic Rankine Cycle. Energies, 10 (619), 1-18.

Patil, R. K., Shende, B. W., and Ghosh, P. K. (1982). Designing a helical-coil heat exchanger. Chemical Engineering, 85-88.

Perry, R. H., and Green, D. (2008). Chemical Engineers Handbook (8th ed.). New York, USA: McGrawHill.

Prabhanjan, D. G., Ragbavan, G. S. V., and Kennic, T. J. (2002). Comparison of heat transfer rates between a straight tube heat exchanger and a helically coiled heat exchanger. lnt. Comm. Heat Mass Transfer, 29 (2), 185-191.

San, S. J.-Y., Chih-Hsiang, H., and Shih-Hao, C. (2012). Heat transfer characteristics of a helical heat exchanger. Applied Thermal Engineering, 39, 114-120.

Sateesha, P., Patel, S., Vavhal, P., and Rahate, H. (2018). Design \& Development of Tube in Tube Helical Coil Heat Exchanger. Int. Res. J. of Science \& Engineering, (Special Issue A2), 245-250.

\section{SEMBLANZA DE LOS AUTORES}

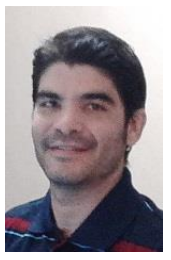

Amaury Pérez Sánchez: Obtuvo el grado de Ingeniero Químico en la Universidad de Camagüey, Cuba, donde actualmente es profesor instructor e investigador auxiliar. En estos momentos se encuentra estudiando un Máster en Biotecnología en el centro de Ingeniería Genética y Biotecnología de Camagüey. Trabaja en líneas de investigación vinculadas fundamentalmente con el diseño de equipos de transferencia de calor y masa, simulación y optimización de procesos y operaciones de la industria química mediante el empleo de simuladores, y la evaluación técnicoeconómica de procesos y plantas biotecnológicas.

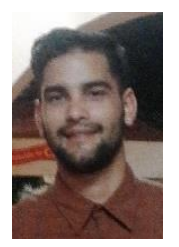

Eddy Javier Pérez Sánchez: Obtuvo el grado de Ingeniero Químico en la Universidad de Camagüey, Cuba. Se desempeña profesionalmente en la Empresa de Cítricos Ceballos, en la provincia de Ciego de Ávila, Cuba, donde atiende la línea de procesamiento de tomate y guayaba para la producción de néctares y pastas. Trabaja en líneas de investigación relacionadas con el diseño, evaluación y/o operación de equipos de transferencia de calor y masa.

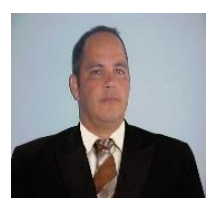

Agustín Heredia Sánchez: Obtuvo el grado de Ingeniero Mecánico en la Universidad de Camagüey, Cuba, en donde se desempeña en la actualidad como especialista de procesos inversionistas. Trabaja en líneas de investigación relacionadas con el diseño mecánico de equipos y sistemas termoenergéticos, así como también la evaluación de equipos y procesos de transferencia de calor y masa.

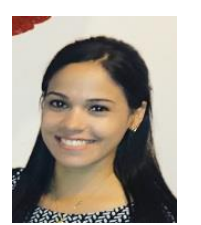

Lorena Pazos Amayuela: Obtuvo el grado de Ingeniero Industrial en la Universidad de Camagüey, Cuba, donde actualmente es profesor instructor e investigadora auxiliar. Su línea de investigación radica fundamentalmente en la planificación, proyección y organización de operaciones y procesos de la industria química, incluyendo la evaluación y diseño de equipos y procesos de la industria química, fundamentalmente aquellos relacionados con la transferencia de calor y masa. 